\title{
Clinical Characteristics, Diagnostic Evaluation, and Antibiotic Prescribing Patterns for Skin Infections in Nursing Homes
}

\author{
Norihiro Yogo ${ }^{1,2 *}$, Gregory Gahm ${ }^{3}$, Bryan C. Knepper, ${ }^{4,5}$, William J. Burman ${ }^{1,2,5,6}$, \\ Philip S. Mehler ${ }^{2,5}$ and Timothy C. Jenkins ${ }^{1,2,5}$ \\ ${ }^{1}$ Department of Medicine, Division of Infectious Diseases, University of Colorado, Aurora, CO, USA, ${ }^{2}$ University of Colorado \\ School of Medicine, Aurora, CO, USA, ${ }^{3}$ Department of Medicine, Division of Geriatrics, University of Colorado, Aurora, CO, \\ USA, ${ }^{4}$ Department of Patient Safety and Quality, Denver Health, Denver, CO, USA, ${ }^{5}$ Denver Health, Denver, CO, USA, \\ ${ }^{6}$ Denver Public Health, Denver Health, Denver, CO, USA
}

OPEN ACCESS

Edited by:

Melissa DePaoli Johnson,

Duke University Medical Center, USA

Reviewed by:

Mark Fagan,

University of Oslo, Norway

Travis Michael Jones,

Duke University Medical Center, USA

*Correspondence: Norihiro Yogo

norihiro.yogo@ucdenver.edu

Specialty section:

This article was submitted

to Infectious Diseases,

a section of the journal

Frontiers in Medicine

Received: 26 January 2016 Accepted: 23 June 2016

Published: 21 July 2016

Citation:

Yogo N, Gahm G, Knepper BC, Burman WJ, Mehler PS and Jenkins TC (2016) Clinical Characteristics, Diagnostic

Evaluation, and Antibiotic Prescribing Patterns for Skin Infections in Nursing Homes.

Front. Med. 3:30.

doi: 10.3389/fmed.2016.00030
Background: The epidemiology and management of skin infections in nursing homes has not been adequately described. We reviewed the characteristics, diagnosis, and treatment of skin infections among residents of nursing homes to identify opportunities to improve antibiotic use.

Methods: This was a retrospective study involving 12 nursing homes in the Denver metropolitan area. For residents at participating nursing homes diagnosed with a skin infection between July 1, 2013 and June 30, 2014, clinical and demographic information was collected through manual chart review.

Results: Of 100 cases included in the study, the most common infections were non-purulent cellulitis $(n=55)$, wound infection $(n=27)$, infected ulcer $(n=8)$, and cutaneous abscess $(n=7)$. In 26 cases, previously published minimum clinical criteria for initiating antibiotics (Loeb criteria) were not met. Most antibiotics $(n=52)$ were initiated as a telephone order following a call from a nurse, and 41 patients were not evaluated by a provider within $48 \mathrm{~h}$ after initiation of antibiotics. Nearly all patients $(n=95)$ were treated with oral antibiotics alone. The median treatment duration was 7 days (interquartile range 7-10); 43 patients received treatment courses of $\geq 10$ days.

Conclusion: Most newly diagnosed skin infections in nursing homes were non-purulent infections treated with oral antibiotics. Antibiotics were initiated by telephone in over half of cases, and lack of a clinical evaluation within $48 \mathrm{~h}$ after starting antibiotics was common. Improved diagnosis through more timely clinical evaluations and decreasing length of therapy are important opportunities for antibiotic stewardship in nursing homes.

Keywords: antimicrobial stewardship, nursing homes, long-term care facilities, skin and soft tissue infection, skin infections 


\section{INTRODUCTION}

Antibiotic resistance and Clostridium difficile infection have been identified as urgent health threats by the Centers for Disease Control and Prevention (1) and have resulted in the need to develop systems to improve antibiotic use as a national priority $(2,3)$. The Infectious Diseases Society of America/Society for Hospital Epidemiology of America (IDSA/SHEA) provide broad recommendations for antimicrobial stewardship interventions (2). These guidelines highlight the importance of improving antibiotic use not only in acute care hospitals but also in nursing homes and long-term care facilities $(2,3)$. Recent nationwide surveys in the United States have demonstrated that $12 \%$ of nursing home residents have an infection at any given time (4), and up to $79 \%$ of nursing home residents receive an antibiotic over the course of a year (5). An estimated $25-75 \%$ of antibiotic use in such facilities is deemed to be inappropriate $(6,7)$. Antibiotic exposure in long-term care facilities is associated with the acquisition of drug-resistant organisms $(8,9)$. Furthermore, there is a markedly increased risk of $C$. difficile infection in the elderly, and an estimated one-quarter of all cases in the United States occur in nursing homes (10). Given that 1.4 million individuals are currently residing in 15,700 nursing homes in the Unites States (11), antimicrobial stewardship in nursing homes is essential to prevent these common complications of antibiotic use.

The most common indications for antibiotics in nursing homes include urinary tract, respiratory tract, and skin and soft tissue infections $(4,12)$. Thus far, studies to improve antibiotic use in nursing homes have focused largely on urinary tract infections (13-15) and pneumonia $(13,16,17)$. In both hospitalized patients and outpatients, skin and soft tissue infections are commonly associated with inappropriate antibiotic use (18-20); however, studies on the diagnosis and management of skin infections in the nursing home setting are lacking. The objectives of this study were to describe the epidemiology, clinical characteristics, diagnostic evaluation, and antibiotic prescribing patterns for skin infections in nursing homes to identify opportunities to improve antibiotic utilization.

\section{MATERIALS AND METHODS}

This was a retrospective study of skin infections involving 12 nursing homes within the Denver metropolitan area between July 1, 2013 and June 30,2014. The 12 participating facilities ranged in size from 60 to 160 patients, with a combined census of 985 patients [818 (83\%) long-term residents]. The patients at these facilities were managed by 67 unique providers: 36 physicians and 31 nurse practitioners or physician assistants. On-site wound care consultation was available at all participating facilities at the discretion of the patient's provider. None of the nursing homes were involved in any other antimicrobial stewardship initiatives either prior to or during the study period. This study was approved by the Colorado Multiple Institutional Review Board.

\section{Case Identification and Data Collection}

Residents identified as having any skin infection in sections I or M of the Minimum Data Set (MDS) (per the facility's usual documentation practices) and initiated on systemic antibiotic therapy were identified for review. Cases involving continuation of therapy from a hospitalization or outside facility, osteoarticular infections, necrotizing soft tissue infections, use of prophylactic or suppressive antibiotic therapy, and existence of a concurrent infection requiring antibiotic therapy were excluded. Cases with unavailable or incomplete medical records were also excluded. For patients who had multiple episodes of a skin infection during the study period, only the initial treatment course was reviewed.

For cases meeting study entry criteria, demographic information, selected comorbid conditions, diagnostic data, antibiotic therapy, and clinical outcomes during a 30-day follow-up period from the start of treatment were manually recorded using a standardized documentation form. Clinical characteristics of the skin infections were determined through review of clinician and nursing documentation. The antibiotic selection and duration were collected through review of nursing home provider order forms and medication administration records.

\section{Cases Classifications and Definitions}

Skin infections were categorized as non-purulent cellulitis, purulent cellulitis, cutaneous abscess, wound infection, or an infected ulcer as documented in provider and/or nursing notes. The Loeb minimum criteria for the initiation of antibiotics for skin infections was defined as new or increasing purulent drainage; or at least two of the clinical features of fever, redness, tenderness, warmth, or new or increased swelling of the affected site (21). Antibiotics were deemed to have been initiated through a call from a nurse if a call to a provider resulted in a verbal order for antibiotics. Antibiotics were considered to be initiated by a non-nursing home provider if they were ordered by a wound care physician, emergency room provider, or at an outside clinic visit. Documentation of an in-person evaluation within $48 \mathrm{~h}$ of the start of antibiotic therapy by any provider - physician, nurse practitioner, physician assistant, or non-nursing home provider - was also recorded. Antibiotics with activity against methicillin-resistant Staphylococcus aureus (MRSA) were defined as tetracyclines, clindamycin, trimethoprim-sulfamethoxazole, vancomycin, linezolid, and daptomycin. Antibiotics with a broadspectrum of Gram-negative activity were defined as $\beta$-lactam $/ \beta$ lactamase inhibitor combinations, second- or third-generation cephalosporins, carbapenems, and fluoroquinolones. Treatment failure was defined as a change in antibiotic regimen or extension of treatment duration due to inadequate clinical response. Relapse within the 30-day follow-up period was defined as recurrence of infection in the same anatomic location after completion of initial therapy. Any adverse events attributed to antibiotics, including $C$. difficile infection, were recorded.

\section{Data Analysis}

The main outcomes of interest included the frequency of the various types of skin infections, the frequency with which antibiotics were started without a clinical evaluation, and antibiotic selection and duration of therapy. Descriptive statistics were calculated for the entire cohort. Given expected differences in the treatment of non-purulent cellulitis as compared with purulent infections 
(abscess, purulent cellulitis, wound infection, and infected ulcers), analyses were also stratified by these two groups. Odds ratios (OR) and 95\% confidence intervals (CI) were used to assess the association with categorical variables. For continuous variables, the Wilcoxon rank-sum test was used. All analyses were performed using SAS version 9.3 (SAS Institute, Cary, NC, USA).

\section{RESULTS}

In total, 135 cases were reviewed. Thirty-five cases were excluded for the following reasons (more than one may have been present): continuation of therapy from an outside institution $(n=13)$, presence of a concurrent infection requiring antibacterial therapy ( $n=12)$, confirmed or suspected osteomyelitis or necrotizing infection $(n=5)$, incomplete medical records $(n=4)$, suppressive or prophylactic antibiotic use $(n=3)$, multiple skin infections during the study period $(n=1)$. The remaining 100 cases were included in the final study population. Demographic and clinical characteristics are shown in Table 1. The most common comorbidities included lymphatic or vascular disease, dementia, and diabetes mellitus.

The most common type of skin infection was non-purulent cellulitis ( $n=55$, Table 2 ); in 8 of these cases, the diagnosis was "bilateral lower extremity cellulitis." Wound infection was the next most common $(n=27)$, of which 19 were postoperative. There were eight cases of infected ulcer, seven cases of cutaneous abscess, and one case of purulent cellulitis. Anatomically, most cases involved the extremities $(n=81), 69$ of which involved the lower extremities. The Loeb minimum criteria for the initiation of antibiotics were not met in 26 patients. Thirty patients had a white blood cell count performed, and 12 had a level $\geq 11,000$ cells/ $\mathrm{mm}^{3}$. Cultures were obtained in only 10 cases, of which 5 yielded a clinically significant isolate; therefore, antibiotic therapy in the vast majority of cases was empiric.

Most antibiotics $(n=52)$ were initiated as a telephone order following a call from a nurse; 41 patients were not evaluated by any provider within $48 \mathrm{~h}$ after initiation of antibiotics. Sixteen patients had their antibiotics initiated by a non-nursing home provider. Fifteen patients had a wound care consultation, and only one patient had an infectious diseases specialist consultation to assist in management. Ninety-five patients were treated with oral

TABLE 1 | Baseline demographic and clinical characteristics.

\begin{tabular}{lc}
\hline & Total cases $\boldsymbol{n}=\mathbf{1 0 0}$ \\
\hline Age (mean, 95\% confidence interval) & $72.2(69.4-75.0)$ \\
Male & 38 \\
Dementia & 41 \\
Diabetes mellitus & 39 \\
Lymphatic or vascular disease $^{\text {a }}$ & 55 \\
Wheelchair-dependence & 54 \\
Bedridden & 9 \\
Hospitalization within 30 days & 37 \\
Skin infection within 30 days & 9 \\
History of MRSA infection and/or colonization & 8 \\
\hline
\end{tabular}

${ }^{a}$ Defined as chronic venous stasis, lymphedema, peripheral arterial disease, or chronic lower extremity edema.

antibiotics only, and five patients received treatment that involved intravenous antibiotics (Table 3). The most common class of antibiotics prescribed was an oral $\beta$-lactam $(n=73) ; 28$ cases were prescribed an oral antibiotic with activity against MRSA; and 18 patients received antibiotics with broad Gram-negative activity.

When comparing the management of non-purulent cellulitis with other infection types, antibiotics with activity against MRSA were used with similar frequency [ 24 vs. $33 \%$, respectively; OR 0.62 (95\% CI: 0.26-1.49)]; however, patients with non-purulent cellulitis were less likely to be prescribed antibiotics with broad Gram-negative activity [9 vs. 31\%; OR 0.22 (95\% CI: 0.07-0.68)]. Overall, the median duration of therapy was 7 days [interquartile range (IQR) 7-10]. In 43 cases, the prescribed duration was $\geq 10$ days. Treatment failure occurred in 15 cases, and 4 patients required admission to an acute care hospital. Relapse within the 30-day follow-up period occurred in 11 cases. An adverse drug event was documented in 11 cases including gastrointestinal side effects in 10 and a rash in one. No patient was diagnosed with C. difficile infection during the follow-up period.

\section{TABLE 2 | Type and clinical features of skin infections.}

Total cases $n=100$

\begin{tabular}{lr}
\hline Type of skin infection $^{\mathbf{a}}$ & 55 \\
Non-purulent cellulitis & 27 \\
Wound infection & \\
Infected ulcer & 7 \\
Cutaneous abscess & \\
Purulent cellulitis & 7 \\
Other skin infection & 7 \\
Recurrence of a prior skin infection & 7
\end{tabular}

Initial location of involvement ${ }^{\mathrm{b}}$

55

27

8

1

7

7
Extremities

Leg

Foot or ankle

Hand or wrist

Arm

Trunk

Abdomen

Buttock/hip

Back

Chest

Head and neck

Groin/inguinal

ypes of wounds associated with skin infection

Postoperative wound

Skin tear or abrasion

Ulcer

Other

Clinical features

Temperature $\geq 100.0^{\circ} \mathrm{F}$

Erythema

Induration

Warmth

Pain

Gross purulence

Absence of Loeb minimum criteria $^{c}$

81
58
11
9
4
15
5
6
4
2
4
2

alncludes five cases with two different types of concurrent skin infection. bIncludes five cases with skin infection in more than one anatomic site. cNew or increasing purulent drainage; or at least two of the clinical features of fever, redness, tenderness, warmth, or new or increased swelling of the affected site (21). 
TABLE 3 | Antibiotic selection, duration of therapy, and clinical outcomes.

Total cases $n=100$

\begin{tabular}{|c|c|}
\hline \multicolumn{2}{|l|}{ Antibiotic selection ${ }^{a}$} \\
\hline Oral $\beta$-lactam & 73 \\
\hline Cephalexin & 65 \\
\hline Amoxicillin/clavulanate & 5 \\
\hline Other oral $\beta$-lactam & 4 \\
\hline Oral anti-MRSA antibiotic & 28 \\
\hline Doxycycline or tetracycline $e^{b}$ & 17 \\
\hline Trimethroprim-sulfamethoxazole & 7 \\
\hline Clindamycin & 5 \\
\hline Fluoroquinolone ${ }^{c}$ & 10 \\
\hline Intravenous antibiotic & 5 \\
\hline Vancomycin & 2 \\
\hline Ertapenem & 2 \\
\hline Ceftriaxone or cefazolin & 2 \\
\hline Antibiotic with broad Gram-negative activity ${ }^{d}$ & 18 \\
\hline Multiple antibiotics prescribed & 18 \\
\hline Sequential therapy & 12 \\
\hline Combination therapy & 6 \\
\hline \multicolumn{2}{|l|}{ Treatment duration } \\
\hline Total duration of therapy, median days (IQR) & $7(7-10)$ \\
\hline Duration of therapy $\geq 10$ days & 43 \\
\hline Duration of therapy $\leq 5$ days & 6 \\
\hline \multicolumn{2}{|l|}{ Clinical outcomes } \\
\hline Treatment failure & 15 \\
\hline Required hospitalization & 4 \\
\hline Discharged to home with antibiotics & 3 \\
\hline \multicolumn{2}{|c|}{$\begin{array}{l}\text { aTotals for subgroups include } 18 \text { patients who received more than } 1 \text { antibiotic. } \\
\text { 'Includes doxycycline use in } 16 \text { cases and tetracycline in } 1 \text { case. } \\
\text { 'Includes levofloxacin use in nine cases and moxifloxacin in one case. } \\
{ }^{d} \text { Defined as } \beta \text {-lactam/ } \beta \text {-lactamase inhibitor combinations, second- or third-generation } \\
\text { cephalosporins, carbapenems, and fluoroquinolones. }\end{array}$} \\
\hline
\end{tabular}

\section{DISCUSSION}

Understanding the clinical characteristics, diagnostic evaluation, and treatment of skin infections in nursing homes is essential to optimize antibiotic use for these common conditions. A number of prior studies have evaluated overall antibiotic prescribing practices in nursing homes and long-term care facilities (22-26); however, to our knowledge, this is the first detailed description of the treatment of skin infections in this population.

The most common type of skin infection in this study was non-purulent cellulitis, accounting for over half of all cases. This contrasts sharply to hospitals and ambulatory care, where a substantially higher proportion of purulent infections has been observed $(19,20)$. Furthermore, in this study, evidence of systemic involvement was uncommon; almost all cases were treated with oral antibiotics alone, and only four cases required hospitalization. These findings suggest that the majority of skin infections in this population were mild, uncomplicated infections.

The diagnosis of skin infections can be challenging. In this study, the most common anatomical location of infection was the lower extremities, a finding that is not surprising since over half of patients had lymphatic or vascular disease and over half were wheelchair dependent. Such vascular insufficiency is frequently associated with conditions that mimic skin infections (e.g., venous stasis dermatitis) and result in misdiagnosis of cellulitis (27). It is notable that eight patients were diagnosed as having "bilateral lower extremity cellulitis," an uncommon clinical phenomenon, which suggests that the diagnosis of cellulitis was inaccurate in some patients. Due to the difficulty of diagnosing infections in the elderly, many of whom may have atypical signs and symptoms of an infection, a set of minimum criteria for the initiation of antibiotics for suspected infection (Loeb criteria) have previously been developed (21). Similar to a previous study, we found that these minimum criteria for skin infection were often not met $(26$ cases in our study), despite the initiation of antibiotic therapy (22). In aggregate, our findings suggest that overdiagnosis of skin infections is likely common and highlight the need for interventions to improve diagnostic accuracy in order to avoid unnecessary antibiotic therapy for conditions that mimic skin infections.

One factor that may have contributed to the apparent overdiagnosis of skin infections in these nursing homes is the lack of timely clinical evaluations, either at the time of or shortly after the initiation of antibiotics. We demonstrated that over half of antibiotic courses were initiated as telephone orders, a finding similar to previous studies, where $54-67 \%$ of antibiotics were initiated over the telephone $(23,24)$. Moreover, nearly half of all patients were not evaluated by a provider within $48 \mathrm{~h}$ of the initiation of antibiotics. This is consistent with prior studies that showed only $44 \%$ of nursing home residents had a physician visit within 1 day of starting antimicrobial therapy (25), $44 \%$ had no documentation of an infection (24), and 56\% did not have a documented physical examination (26). Clearly, in order to improve antibiotic use in nursing homes, processes that promote timely clinical evaluations to increase the accuracy of initial diagnosis and allow monitoring of response to antibiotic therapy are essential.

Overall, $95 \%$ of patients were treated in the absence of microbiological data to guide antibiotic therapy. This is likely due to the fact that most cases were non-purulent cellulitis, which is most often not able to be cultured; in contrast, purulent infections, such as abscesses, were relatively uncommon. Consistent with national guideline recommendations for the treatment of non-purulent cellulitis (28), the most commonly used antibiotics in this study were oral $\beta$-lactams. The use of antibiotics with activity against MRSA was less common ( $<30 \%$ of cases), and notably, there was no difference in use between non-purulent cellulitis and purulent infections where MRSA is common, such as abscesses, purulent cellulitis, wound infections, and infected ulcers. This suggests that the risk for MRSA was not a determinant in choosing an antibiotic regimen, and thus, antibiotic use could be improved through promoting appropriate use of MRSA-active agents in purulent infections. Likewise, 18 patients received broad-spectrum Gramnegative therapy, and 18 were treated with multiple antibiotics. Since national guidelines recommend the use of a single agent targeted toward Gram-positive pathogens (28), there may be an opportunity to reduce use of overly broad-spectrum antibiotic regimens in nursing home residents with skin infections. Although the median duration of antibiotic therapy was 7 days, over $40 \%$ of patients were treated for 10 days or longer. This is of importance since a treatment course of 5 days is currently recommended for uncomplicated skin infections (28); therefore, shortening treatment durations represent another important opportunity to reduce antibiotic exposure. 
Nursing homes provide unique challenges and barriers to optimize antibiotic use, such as clinical providers being located off-site (6). This highlights the need for interventions to be specifically tailored for the nursing home setting to overcome these obstacles in order to be successful $(2,6)$. Effective interventions studied to date have included the implementation of practice guidelines $(13,14,16,17)$, clinician education (29), regular infectious diseases specialist consultation (30), and prospective audit with prescribing recommendations via telemedicine (31). It is notable that each of these interventions could potentially be applied - alone or in combination - to improve the management of skin and soft tissue infections in nursing homes. This type of infection-specific approach to antibiotic stewardship has been advocated by the CDC (32). Given the findings of this study, we believe that future nursing home interventions to reduce antibiotic overuse for skin and soft tissue infections need to incorporate early clinical evaluations to be most effective.

Our study has several important limitations. First, we retrospectively identified cases at each facility using the MDS, and as such, this study may not have captured every skin infection that occurred over the study period. Indeed, the limited number of included cases in this study suggests that the use of MDS was not likely a comprehensive surveillance method for new skin infections. Second, the retrospective study design with reliance on medical record documentation may have led to misclassification of cases or incomplete information. Likewise, applying the Loeb minimum criteria for this study was subject to the accuracy in assessment and documentation of the nursing home staff. Third, the study involved nursing homes in the Denver metropolitan area only; this may limit the generalizability of our findings, since local antimicrobial susceptibility patterns and antibiotic prescribing behaviors may differ elsewhere. Lastly, the small sample size did not allow for individual subgroup analysis for the different types of skin infections.

In summary, most skin infections in nursing homes appeared to be relatively mild cases of non-purulent cellulitis. Over half

\section{REFERENCES}

1. Centers for Disease Control and Prevention. Antibiotic Resistance Threats in the United States, 2013. Atlanta: CDC (2013). Available from: http://www.cdc. gov/drugresistance/threat-report-2013/pdf/ar-threats-2013-508.pdf

2. Barlam TF, Cosgrove SE, Abbo LM, MacDougall C, Schuetz AN, Septimus EJ, et al. Implementing an antibiotic stewardship program: guidelines by the Infectious Diseases Society of America and the Society for Healthcare Epidemiology of America. Clin Infect Dis (2016) 62(10):e51-77. doi:10.1093/ cid/ciw118

3. The White House. National Action Plan for Combating Antibiotic-Resistant Bacteria. (2015). Available from: https://www.whitehouse.gov/sites/default/files/ docs/national_action_plan_for_combating_antibotic-resistant_bacteria.pdf

4. Dwyer LL, Harris-Kojetin LD, Valverde RH, Frazier JM, Simon AE, Stone $\mathrm{ND}$, et al. Infections in long-term care populations in the United States. J Am Geriatr Soc (2013) 61:341-9. doi:10.1111/jgs.12153

5. van Buul LV, van der Steen JT, Veenhuizen RB, Achterberg WP, Schellevis FG, Essink RT, et al. Antibiotic use and resistance in long term care facilities. J Am Med Dir Assoc (2012) 13(6):.e1-13. doi:10.1016/j.jamda.2012. 04.004

6. Rhee SM, Stone ND. Antimicrobial stewardship in long-term care facilities. Infect Dis Clin N Am (2014) 28:237-46. doi:10.1016/j.idc.2014.01.001 of all antibiotics were initiated over the telephone after a call from a nurse, nearly half of all cases had no clinical assessment by a provider within $48 \mathrm{~h}$ after initiation of antibiotics, and over one-quarter of cases did not meet the Loeb minimum criteria for the initiation of antibiotics. Antibiotic therapy was frequently broader in spectrum and more prolonged than recommended in national guidelines. Our findings suggest that skin infections represent an important opportunity for antibiotic stewardship in nursing homes and that prescribing could be improved through interventions that improve the accuracy of diagnosis, facilitate more timely clinical evaluations, and promote shorter courses of antibiotic therapy with Gram-positive activity.

\section{AUTHOR CONTRIBUTIONS}

NY is the lead investigator and headed study design, data collection, and primary authorship of the manuscript. GG is the medical director at each participating facility and participated in study design, facilitated access to medical records, and participated in manuscript review. BK is the primary statistician and helped with study design, statistical analysis, and participated in manuscript review. WB and PM are coinvestigators and participated in study design and manuscript review. TJ is the senior mentor of the study and oversaw study design, participated in statistical analysis, and is the primary editor of the manuscript.

\section{ACKNOWLEDGMENTS}

We are grateful to the staff at each of the participating nursing homes for facilitating the chart review.

\section{FUNDING}

This work was funded in part by the Department of Patient Safety and Quality, Denver Health. Dr. TJ was supported by the National Institute of Allergy and Infectious Diseases, National Institute of Health (TJ: K23 AI099082).

7. Nicolle LE, Bentley DW, Garibaldi R, Neuhaus EG, Smith PW. Antimicrobial use in long-term-care facilities. Infect Control Hosp Epidemiol (2000) 21(8):537-45. doi:10.1086/501798

8. O'Fallon E, Kandel R, Schreiber R, D'Agata EM. Acquisition of multidrug-resistant Gram-negative bacteria: incidence and risk factors within a long-term care population. Infect Control Hosp Epidemiol (2010) 31(11):1148-53. doi:10.1086/656590

9. Tinelli M, Cataldo MA, Mantengoli E, Cadeddu C, Cunietti E, Luzzaro F, et al. Epidemiology and genetic characteristics of extended-spectrum B-lactamase producing Gram-negative bacteria causing urinary tract infections in long-term care facilities. J Antimicrob Chemother (2012) 67(12):2982-7. doi:10.1093/jac/dks300

10. Lessa FC, Mu Y, Bamberg WM, Beldavs ZG, Dumyati GK, Dunn JR, et al. Burden of Clostridium difficile infection in the United States. $N$ Engl J Med (2015) 372(9):825-34. doi:10.1056/NEJMoa1408913

11. Harris-Kojetin L, Sengupta M, Park-Lee E, Valverde R. Long-term Care Services in the United States: 2013 Overview. National Health Care Statistics Reports; No 1. Hyattsville, MD: National Center for Health Statistics (2013). Available from: http://www.cdc.gov/nchs/data/nsltcp/long_term_care_services_2013.pdf

12. Benoit SR, Nsa W, Richards CL, Bratzler DW, Shefer AM, Steele LM, et al. Factors associated with antimicrobial use in nursing homes: a multilevel model. J Am Geriatr Soc (2008) 56:2039-44. doi:10.1111/j.1532-5415.2008.01967.x 
13. Nicolle LE. Antimicrobial stewardship in long term care facilities: what is effective? Antimicrob Resist Infect Control. (2014) 3(1):6. doi:10.1186/20472994-3-6

14. Loeb M, Brazil K, Lohfeld L, McGeer A, Simor A, Stevenson K, et al. Effect of a multifaceted intervention on number of antimicrobial prescriptions for suspected urinary tract infections in residents of nursing homes: cluster randomised controlled trial. BMJ (2005) 331(7518):669. doi:10.1136/ bmj.38602.586343.55

15. Juthani-Mehta M, Quagliarello V, Perrelli E, Towle V, Van Ness PH, Tinetti M. Clinical features to identify urinary tract infections in nursing home residents: a cohort study. JAm Geriatr Soc (2009) 57(6):963-70. doi:10.1111/j.1532-5415.2009.02227.x

16. Linnebur SA, Fish DN, Rusein JM, Radcliff TA, Oman KS, Fink R, et al. Impact of a multidisciplinary intervention in antibiotic use for nursing home-acquired pneumonia. Am J Geriatr Pharmacother (2011) 3:442-51. doi:10.1016/j.amjopharm.2011.09.009

17. Naughton BJ, Mylotte JM, Ramadan F, Karuza J, Priore RL. Antibiotic use, hospital admissions, and mortality before and after implementing guidelines for nursing home-acquired pneumonia. J Am Geriatr Soc (2001) 3:1020-4. doi:10.1046/j.1532-5415.2001.49203.x

18. Jenkins TC, Sabel AL, Sarcone EE, Price CS, Mehler PS, Burman WJ. Skin and soft-tissue infections requiring hospitalization at an academic medical center: opportunities for antimicrobial stewardship. Clin Infect Dis (2010) 51(8):895-903. doi:10.1086/656431

19. Jenkins TC, Knepper BC, Moore SJ, O'Leary ST, Brooke C, Saveli CC, et al. Antibiotic prescribing practices in a multicenter cohort of patients hospitalized for acute bacterial skin and skin structure infection. Infect Control Hosp Epidemiol (2014) 35(10):1241-50. doi:10.1086/678056

20. Hurley HJ, Knepper BC, Price CS, Mehler PS, Burman WJ, Jenkins TC. Avoidable antibiotic exposure for uncomplicated skin and soft tissue infections in the ambulatory care setting. Am J Med (2013) 126(12):1099-106. doi:10.1016/j.amjmed.2013.08.016

21. Loeb M, Bentley DW, Bradley S, Crossley K, Garibaldi R, Gantz N, et al. Development of minimum criteria for the initiation of antibiotics in residents of long-term-care facilities: results of a consensus conference. Infect Control Hosp Epidemiol (2001) 22(2):120-4. doi:10.1086/501875

22. Olsho LE, Bertrand RM, Edwards AS, Hadden LS, Morefield GB, Hurd D, et al. Does adherence to the Loeb minimum criteria reduce antibiotic prescribing rates in nursing homes? J Am Med Dir Assoc (2013) 14(4):.e1-7. doi:10.1016/j. jamda.2013.01.002

23. Katz PR, Beam TR Jr, Brand F, Boyce K. Antibiotic use in the nursing home. Physician practice patterns. Arch Intern Med (1990) 150:1465-8. doi:10.1001/ archinte.150.7.1465
24. Richards CL Jr, Darradji M, Weinberg A, Ouslander JG. Antimicrobial use in post-acute care: a retrospective descriptive analysis in seven long-term care facilities in Georgia. J Am Med Dir Assoc (2005) 6:109-12. doi:10.1016/j. jamda.2004.12.021

25. Daneman N, Gruneir A, Newman A, Fischer HD, Bronskill SE, Rochon PA, et al. Antibiotic use in long-term care facilities. J Antimicrob Chemother (2011) 66:2856-63. doi:10.1093/jac/dkr395

26. Warren JW, Palumbo FB, Fitterman L, Speedie SM. Incidence and characteristics of antibiotic use in nursing home patients. J Am Ger Soc (1991) 39(10):963-72. doi:10.1111/j.1532-5415.1991.tb04042.x

27. David CV, Chira S, Eells SJ, Ladrigan M, Papier A, Miller LG, et al. Diagnostic accuracy in patients admitted to hospitals with cellulitis. Dermatol Online J (2011) 17(3):1.

28. Stevens DL, Bisno AL, Chambers HF, Dellinger EP, Goldstein EJ, Gorbach SL, et al. Practice guidelines for the diagnosis and management of skin and soft tissue infections: 2014 update by the infectious diseases society of America. Clin Infect Dis (2014) 59(2):147-59. doi:10.1093/cid/ciu444

29. Monette J, Miller MA, Monette M, Laurier C, Boivin JF, Sourial N, et al. Effect of an educational intervention on optimizing antibiotic prescribing in long-term care facilities. J Am Ger Soc (2007) 55:1231-5. doi:10.1111/j.15325415.2007.01250.x

30. Jump RL, Olds DM, Seifi N, Kypriotakis G, Jury LA, Peron EP, et al. Effective antimicrobial stewardship in a long-term care facility through an infectious disease consultation service: keeping a LID on antibiotic use. Infect Control Hosp Epidemiol (2012) 33(12):1185-92. doi:10.1086/668429

31. Beaulac K, Corcione S, Epstein L, Davidson LE, Doron S. Antimicrobial stewardship in a long-term acute care hospital using offsite electronic medical record audit. Infect Control Hosp Epidemiol (2016) 37(4):433-9. doi:10.1017/ ice.2015.319

32. Pollack LA, Srinivasan A. Core elements of hospital antibiotic stewardship programs from the Centers for Disease Control and Prevention. Clin Infect Dis (2014) 59(Suppl 3):S97-100. doi:10.1093/cid/ciu542

Conflict of Interest Statement: The authors declare that the research was conducted in the absence of any commercial or financial relationships that could be construed as a potential conflict of interest.

Copyright (c) 2016 Yogo, Gahm, Knepper, Burman, Mehler and Jenkins. This is an open-access article distributed under the terms of the Creative Commons Attribution License (CC BY). The use, distribution or reproduction in other forums is permitted, provided the original author(s) or licensor are credited and that the original publication in this journal is cited, in accordance with accepted academic practice. No use, distribution or reproduction is permitted which does not comply with these terms. 